\title{
Unusual Electron Transport Properties of $\mathrm{U}_{0.05} \mathrm{Y}_{0.95} \mathrm{Al}_{2}$
}

\author{
V.H. TRAN \\ Institute of Low Temperature and Structure Research \\ Polish Academy of Sciences \\ P.O. Box 1410, 50-950 Wrocław, Poland
}

\begin{abstract}
We report a detailed study of the resistivity $(\rho(T))$ and thermoelectric power $(S(T))$ of the alloy $\mathrm{U}_{0.05} \mathrm{Y}_{0.95} \mathrm{Al}_{2}$, which crystallises in the cubic $\mathrm{MgCu}_{2}$-type structure. The resistivity is found to follow $\rho(T) \propto \ln T$ between 15 and $40 \mathrm{~K}$ and a quasi-linear temperature dependence $-T^{1.16}$ for $T<10 \mathrm{~K}$. At a magnetic field of $9 \mathrm{~T}$ a Kondo-type maximum occurs in the resistivity data at $7 \mathrm{~K}$, below which the Fermi liquid behaviour $\left(\rho(T) \propto T^{2}\right)$ is recovered. $S(T) / T$ shows a $T^{0.5}$ power law for $T<12 \mathrm{~K}$. The low-temperature unconventional features observed in $\mathrm{U}_{0.05} \mathrm{Y}_{0.95} \mathrm{Al}_{2}$ are presumably due to the presence of the two-channel Kondo effect.
\end{abstract}

PACS numbers: 72.15.Jf, 72.15.Qm

\section{Introduction}

The non-Fermi liquid (NFL) ground state in strongly correlated electron systems, which are subjects of intensive theoretical and experimental studies [1,2], may have different physical origins. On one hand, a number of NFL systems have been suggested to locate near to a quantum critical point at $T=0 \mathrm{~K}$. The NFL properties of these systems in general are caused by fluctuations of an order parameter in the vicinity of second-order phase transition. On the other hand, NFL behaviour observed in diluted $f$-electron systems has been attributed to connect with some kind of disorder. In the latter systems, multichannel Kondo effect or distribution of the Kondo temperatures are possible sources of NFL behaviour.

The evolution of non-Fermi liquid state in the solid solutions $\mathrm{U}_{1-x} \mathrm{Y}_{x} \mathrm{Al}_{2}$ is interesting, since the NFL feature appears for $x=0.85-0.95$ when spin fluctuations present in $\mathrm{UAl}_{2}$ become suppressed [3]. However, the role of crystallographic disorder seems also to rely on an introduction of spin glass feature, and therefore the microscopic mechanism behind the NFL behaviour is not fully clarified yet. In the present work, we report a detailed study of electron transport properties of $\mathrm{U}_{0.05} \mathrm{Y}_{0.95} \mathrm{Al}_{2}$. We hope that the data presented in this contribution will give new insight into the nature of the NFL state observed in this alloy. 


\section{Results and discussion}

Polycrystalline sample of $\mathrm{U}_{0.05} \mathrm{Y}_{0.95} \mathrm{Al}_{2}$ was prepared by arc melting stoichiometric amounts of high-quality metals. The sample after annealing for one week at $650^{\circ}$ was checked by X-ray diffraction and electron microprobe analysis. All reflections in the room-temperature $\mathrm{X}$-ray diffraction pattern were indexed to belong to the proper cubic $\mathrm{MgCu}_{2}$-type structure (space group $F d 3 m$ ). The lattice parameter $a$ of the studied sample was refined to be $7.859(3) \AA$, practically the same as was previously reported [3].

Figure 1a shows the temperature dependence of the electrical resistivity for $\mathrm{U}_{0.05} \mathrm{Y}_{0.95} \mathrm{Al}_{2}$. The measurements were performed using a standard four-probe technique with excitation currents of $10 \mathrm{~mA}$ and frequency of $37 \mathrm{~Hz}$. It has a weak temperature dependence, suggesting an influence of atomic disorder. Furthermore, an occurrence of minimum at about $T_{\min }=60 \mathrm{~K}$ and visible $\ln T$ dependence (dashed line in Fig. 1a) below $T_{\min }$ imply the presence of the Kondo effect in this alloy. Assuming the Kondo temperature $\left(T_{\mathrm{K}}\right)$ to be the point, at which the resistivity data deviate from logarithmic behaviour, one estimates $T_{\mathrm{K}}=15 \mathrm{~K}$ for $\mathrm{U}_{0.05} \mathrm{Y}_{0.95} \mathrm{Al}_{2}$. For temperatures below $10 \mathrm{~K}$, the data can be fitted by power law dependence $\rho(T) \sim T^{n}$ with $n=1.16( \pm 0.08)$, and the power law behaviour is demonstrated as solid line in Fig. 1a. It is worthwhile to recall that the power law exponent $n$ ranging from 1 to 1.5 was predicted by Rosch [4]. The author has considered the interplay of disorder and spin fluctuations near a quantum critical point, where magnetism has just been suppressed to $T=0 \mathrm{~K}$. On the other hand, the value $n=1$ is commonly found for other NFL diluted systems, for instance, $\mathrm{U}_{1-x} \mathrm{Y}_{x} \mathrm{Pd}_{3}$ [5], $\mathrm{U}_{1-x} \mathrm{Th}_{x} \mathrm{Pd}_{2} \mathrm{Al}_{3}$ [6], $\mathrm{U}_{1-x} \mathrm{Y}_{x} \mathrm{Pd}_{2} \mathrm{Al}_{3}$ [7] and $\left(\mathrm{La}_{1-x} \mathrm{U}_{x}\right)_{2} \mathrm{Zn}_{17}$ [8]. For these solid solutions, the two-channel Kondo model was postulated, though there exists discrepancy with the theory, which predicts $n=0.5$.

The electrical resistivity measured in magnetic fields up to $9 \mathrm{~T}$ is displayed in the inset of Fig. 1a. It is clear that the data in fields up to $1 \mathrm{~T}$ show a similar behaviour as that at $0 \mathrm{~T}$. Interestingly, the resistivity curves show a change of curvature at fields between 3 and $5 \mathrm{~T}$, from the negative derivative $\mathrm{d} \rho / \mathrm{d} T$ to positive one. A detailed analysis of the data at 7 and $9 \mathrm{~T}$ revealed the development of maximum located at $7 \mathrm{~K}$. The appearance of this maximum is expected for the Kondo systems, since the magnetic fields reduce the Kondo interactions and then the maximum is a result of competitive interactions between the Kondo and phonon contributions. This maximum together with the observation of a $\rho(T) \propto T^{2}$ dependence below $4 \mathrm{~K}$ for the data collected at $9 \mathrm{~T}$ stress the recovering of the Fermi liquid behaviour by the applied magnetic fields.

In Fig. 1b we show the thermoelectric power of $\mathrm{U}_{0.05} \mathrm{Y}_{0.95} \mathrm{Al}_{2}$ measured by a differential method. The alloy displays a Kondo-like increase at elevated temperature range and a positive maximum of about $13 \mu \mathrm{V} / \mathrm{K}$ at $T_{\max }=42 \mathrm{~K}$. It should be mentioned that a similar $S(T)$ behaviour has been observed for a number of Ce-based Kondo systems [9], and can be explained with the help of the single- 

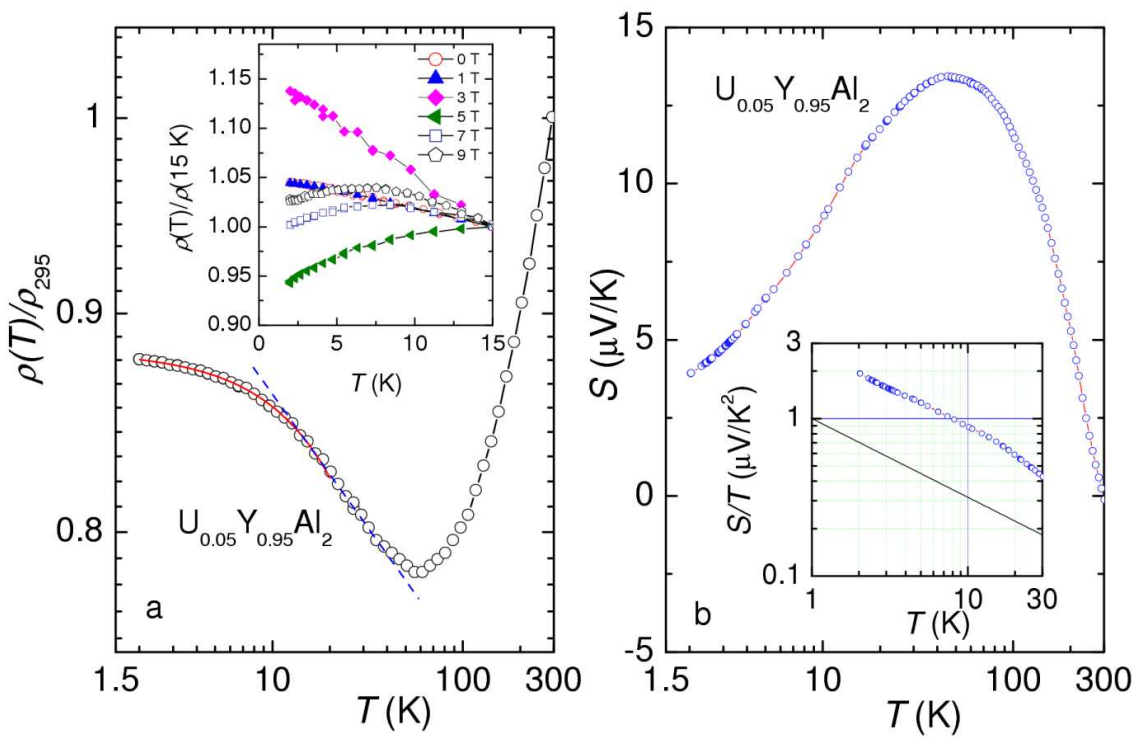

Fig. 1. (a) Electrical resistivity of $\mathrm{U}_{0.05} \mathrm{Y}_{0.95} \mathrm{Al}_{2}$. Inset: the $\rho(T)$ data in several magnetic fields. Let us note that the data were normalised to value at $15 \mathrm{~K}$. (b) Thermoelectric power $S(T)$ of $\mathrm{U}_{0.05} \mathrm{Y}_{0.95} \mathrm{Al}_{2}$. The inset shows a power $T^{1 / 2}$ law behaviour.

-impurity Kondo effect model [10,11]. Accordingly to the model, a maximum with large magnitude is expected to locate around the characteristic temperature of the order of the Kondo temperature $T_{\mathrm{K}}$. A remarkable feature of $\mathrm{U}_{0.05} \mathrm{Y}_{0.95} \mathrm{Al}_{2}$ is its low-temperature behaviour (see inset of Fig. 1b). Below $10 \mathrm{~K}$, the $S(T) / T$ curve is well described by a power $T^{1 / 2}$ law, consistent with predictions of the two-channel Kondo effect [12]. This dependence may be associated with the unconventional behaviour observed previously in the specific heat [3]. Nevertheless, one may recall that there are few experimental reports on thermoelectric power of NFL systems [13-15], but none of them does not show a power $T^{1 / 2}$ law expected for the twochannel Kondo effect yet.

In summary, we have investigated a sample of $\mathrm{U}_{0.05} \mathrm{Y}_{0.95} \mathrm{Al}_{2}$ by means of electrical resistivity and thermoelectric power measurements. The studied physical properties exhibit several interesting features: (i) The resistivity shows a quasi-linear temperature dependence below $10 \mathrm{~K}$ and the Kondo-type minimum at $60 \mathrm{~K}$. (ii) In the magnetic field range of $3-5 \mathrm{~T}$ the $\rho(T)$ curves change curvatures and above $7 \mathrm{~T}$ the Fermi liquid behaviour with a coherence-like maximum and $T^{2}$ dependence recovers. (iii) The low-temperature thermoelectric power $S(T) / T$ follows a $T^{1 / 2}$ power law. Thus the measured electron transport properties may support the NFL state previously observed in $\mathrm{U}_{0.05} \mathrm{Y}_{0.95} \mathrm{Al}_{2}$. Moreover, our data tend to argue an interpretation in terms of the two-channel Kondo scenario. 


\section{Acknowledgments}

This work is supported by the grant No. N202 082 31/0449 from thr Ministry of Science and Higher Education in Poland.

\section{References}

[1] G.R. Stewart, Rev. Mod. Phys. 73, 797 (2001); 78, 743 (2006).

[2] E. Miranda, V. Bobrosavljević, Rep. Prog. Phys. 68, 2337 (2005).

[3] F. Mayr, G.-F. v. Blanckenhagen, G.R. Stewart, Phys. Rev. B 55, 947 (1997).

[4] A. Rosch, Phys. Rev. Lett. 82, 4280 (1999).

[5] C.L. Seaman, M.B. Maple, Physica B 199-200, 396 (1994).

[6] R.P. Dickey, A. Amann, E.J. Freeman, M.C. de Andrade, M.P. Maple, Phys. Rev. B 62, 3979 (2000).

[7] E.J. Freeman, M.C. de Andrade, R.P. Dickey, N.R. Dilley, M.B. Maple, Phys. Rev. B 58, 16027 (1998).

[8] G.-F. von Blanckenhagen, E.-W. Scheidt, T. Schreiner, G.R. Stewart, Phys. Rev. B 64, 064413 (2001).

[9] D. Jaccard, A. Basset, J. Sierro, J. Pierre, J. Low. Temp. Phys. 80, 285 (1990).

[10] D. Cox, N. Grewe, Z. Phys. B, Condens. Matter 71, 321 (1988).

[11] N.E. Bickers, D.L. Cox, J.W. Wilkins, Phys. Rev. Lett. 54, 230 (1985).

[12] D.L. Cox, unpublished results.

[13] J. McCarten, S.E. Brown, C.L. Seaman, M.P. Maple, Phys. Rev. B 49, 6400 (1994).

[14] H. Sato, J. Urakawa, Y. Aoki, T. Namiki, H. Sugawara, Y. Onuki, H. Amitsuka, T. Sakakibara, Physica B 259-261, 390 (1999).

[15] G.F. Chen, I. Sakamoto, S. Ohara, T. Takami, H. Ikuta, U. Mizutami, Phys. Rev. $B$ 69, 014420 (2004). 